\title{
Design and the Reliability Research of the Restorable Dental Implant System Aiming at the Central Screw Fracture
}

\author{
Xiao Zhang ${ }^{1, \mathrm{a}}$,Lei Liu ${ }^{{ }^{1}, 2, \mathrm{~b}}$, Xian-shuai Chen ${ }^{1,3, \mathrm{c}}$, Wei Feng ${ }^{1, \mathrm{~d}}$, Yang Chen ${ }^{4, e}$ \\ ${ }^{1}$ Shenzhen Institutes of Advanced Technology, Shenzhen, China, 518055; \\ ${ }^{2}$ Guangzhou Janus Biotechnology Co.,Ltd., Guangzhou, China, 511458; \\ ${ }^{3}$ Foshan Angels Biotechnology Co.,Ltd., Foshan, China, 528000; \\ ${ }^{4}$ Shenzhen Second People's Hospital, Shenzhen, China, 518000; \\ azx623@126.com, b995724151@qq.com, cxs.chen@gzjanus.com, ${ }^{\text {d2 } 283775186 @ q q . c o m, ~}$ \\ e1342225029@qq.com
}

KeyWords: Central Screw Fracture, Mechanical Analysis, Restorable Implant System, Fatigue Analysis

\begin{abstract}
In the modern dental surgery, due to the misoperation during the surgery or the higher loading force suffered for long time, the situation of the central screw fracture usually occurs, and thus which may result in the failure of the surgery or of implant prosthesis; and aiming at this fact, we now designed a kind of dental implant system which may satisfy the requirement of convenient restoration after the central screw factures. The advantage of this dental implant system was that, after the central screw facture occurs, we can replace the central screw for restoration directly without needing to take out the centre screw which had broken within the dental implant. By means of the finite element analysis, we can get to know that, if a torque equal to 30N.CM is inflicted on the original central screw, the dental implant system may produce a maximum equivalent stress equal to 828.49MPA while if a torque equal to $60 \mathrm{~N} . \mathrm{cm}$ is inflicted on the central screw used for restoration, the dental implant system may produce a maximum equivalent stress equal to 526.44MPA. Thus the torsional property of the dental implant system after restoration was improved significantly on the contrary. Furthermore, we analyzed the occlusal force and the fatigue aimed at the dental implant system after restoration revealed, and we analyzed the mechanics and the fatigue status of the dental implant system and the osseous tissue by means of inflicting a force to the dental crown, which was equal to $300 \mathrm{~N}$ and the angel between which and the upright direct was equal to $0,15^{\circ}, 30^{\circ}$ and $45^{\circ}$ respectively under the implantation status in the horizontal bone and implantation status in the stimulated osteanabrosis of $2 \mathrm{~mm}$. The above analysis revealed that, the maximum equivalent stress produced by the restorable implant system was lower than its yield strength, and the minimum safety factor was higher than 1 . Furthermore, this type of dental implant system has superior safety, and it is convenient for restoration and fit for the modern dentistry implant surgery, thus it has a giant application potential.
\end{abstract}

\section{Introduction}

In the modern dental restoration surgery, the restoration by means of implantation has been the mainstream way of surgery; and the dental implant system has been applied to the dentition defect and the dentition defect restoration based on the masticatory function greatly superior to the other conventional false tooth, and the advantages such as beauty, comfort, and its property of stable and reliable for a long time and thus is referred to as 'the third set of teeth'. [1-2].

The dental implant system mainly consists of the implant body, the abutment and the central screw. In the final restoration, the implant body may be implanted into the alveolar bone, the abutment may cooperate to the implant body, and bonds the dental crown may be bonded on the abutment by means of the central screw fixation. So that the reliability of the dental implant system may be one of the key factors for the successful dental restoration; furthermore, during assembling the central screw in the 
course of surgery, the too high twisting force or the positional deviation in the assembling may lead to that the central screw will bear the too high occlusal loading which may result in the central screw fracture, this was the common case of failure implantation restoration. It is more important that, once the central screw fracture, the restoration difficulty of the fractured central screw in the later stage will increase, and which main lies in the difficulty of eradicating the fractured central screw; all of the current restoration ways were rotating it out by means of shocking using the forceps or polishing the location of the tool again. However, the eradication may be performed only within the mouth with small space, and the rather complex eradication procedure is easy to damage the import body and the synosteosis. The author of the present article designed a restorable dental implant system compatible with two types of central screw simultaneously aimming at the situation of high difficulty of restoration. The components comprises an additional central screw compared to the coventional dental implant system, and when the original central screw fractures, the physician may replace it with the central screw for restoration and finish the restoration without needing to take out the fractured central screw, the operation of this type restoration is simple and the dental implant system after restoration may acquire perfect biological mechanical property and reliability. The Fig. 1 presented the schematic diagram of the restorable dental implant system implanted in the alveolar bone, and the difference between that and the common popular type of dental implant system only lied in addition of a restorable central screw..

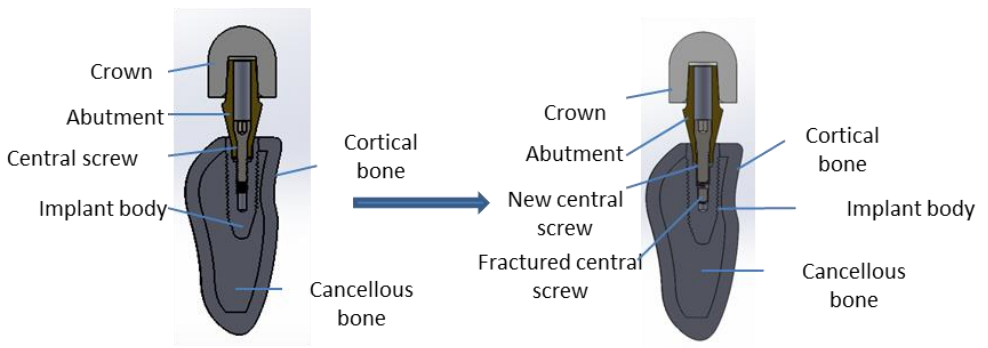

(a) is the original dental implant system (b ) is the restorable dental implant system aimed at the central screw fracture, which is abbreviated restorable dental implant system

Fig. 1 Schematic diagram of the restorable dental implant system model implanted into the alveolar bone

In this article, we designed and established the models for the general type dental implant system and the restorable dental implant system by means of the CAD software SolidWorks, and performed the performance analysis before and after restoration using the software of finite element analysis, and first inflicted the torques to the original central screw and the central screw after restoration, and perform the comparative analysis on the torsional properties of the dental implant systems. Furthermore, we performed the analysis on the biomechanics and the fatigue and studied the stress distribution of the dental implant system and the osseous tissue by means of loading the higher occlusal force equal to $300 \mathrm{~N}$ and the angel between which and the upright direct were $0^{\circ}, 15^{\circ}, 30^{\circ}$ and $45^{\circ}$ respectively on the dental crown under the conditions of the implantion on horizontal bone and the implantation on the osteanabrosis of $2 \mathrm{~mm}$, and we also performed analysis on the cyclic fatigue properties of the denta implant system at the bearing direction of the long time occlusion.

\section{Dental implant system design}

The restorable dental implant system designed in the present research mainly comprised the implant body, the abutment, the central screw, and the restorable central screw based on the design philosophy of the positioning using the inner hexagonal corner, the platform shifting, the Morse's taper, the self-drill property and the double-taper thread. Wherein the taper of the inner connecting cone of the implant body was equal to $3^{\circ}$ and was used for the tight junction of the implant body and the abutment. The outer screw of the implant body was designed as the trapezoidal thread of which the screw pitch $\mathrm{P}$ was $0.6 \mathrm{~mm}$, the diameter and the length were $5 \mathrm{~mm}$ and $15 \mathrm{~mm}$ respectively, and 
which was mainly used for restoring the molar, and the threads of the central screws and the restorable central screws were M1.6 and M2 respectively, and for the inner thread hole of the implant body was designed as a ladder type of which the upper part may be used for coordinating with the reserved restorable screw and the lower part may be used for coordinating to the conventional central screw. The abutment had a diameter equal to $5 \mathrm{~mm}$, of which the inner thread of the outer hexagonal part was used for coordinating with the restorable central screw to enhance the connecting strength of the dental implant system; the abutment had a height equal to $6.5 \mathrm{~mm}$, and the implant body was positioned to the abutment through the hexagon, where the central screw may impact the connecting cone, thus it may acquire the cold welding type connecting effect and may prevent the bacterial microleakage.

The restorable central screw had the length equal to $9 \mathrm{~mm}$, and the thread specification of M2X0.4. The surface of the dental crown bearing the main loading was cohered on the abutment. The stimulated dental crown adopted the way of the combing a cylinder of which the diameter and the height were $10 \mathrm{~mm}$ and $5 \mathrm{~mm}$ respectively and a hemispheric of which the radium was $5 \mathrm{~mm}$. and the alveolar bone model was designed based on the data of phatnoma tissue in the human acquired in the CT scanning. The osseous tissue comprised two parts of the cortical bone and the cancellous bone, where the cortical bone within the thickness of $2 \mathrm{~mm}$ wrapped the outer side of the cancellous bone [4]。We established the three-dimensional models of the implant body, the abutment, the central screw. the restorable central screw, the dental crown, the cortical bone and the cancellous bone respectively using the SolidWorks software based on the above parameters. The characterics of the models for the restorable dental implant system and the osseous tissue were shown in the Fig. 2.

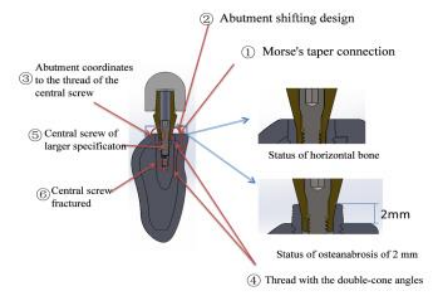

Fig. 2 Characterics of the restorable dental implant system

\section{Finite element analysis}

\subsection{Materials and methods}

We performed the analysis on the static force and dynamic fatigue by means of importing the file into the ANSYS software of finite element analysis through the CAD interface of the ANSYS software. By means of meshing the intelligent size by means of the three-dimensional entity unit of tetrahedron We also established the three-dimensional finite element model for the restorable dental implant system-osseous tissue, which was shown in Fig. 3.

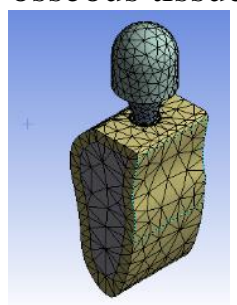

Fig.3 Performance analysis model of the restorable dental implant system

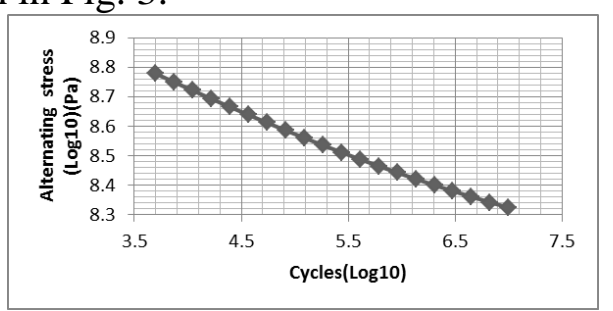

Fig.4 S-N curve for Ti6A14V

All of the tissues and the material in the hypothesis model of the present research were the continuous, homogeneous and isotropous, linear, and elastic material, and all of the material in the dental implant system were titanium alloy Ti6Al4V, and the material of the dental crown was zirconium oxide The performance data were shown in table 1, and the $\mathrm{S}-\mathrm{N}$ curve for Ti6A14V was shown as Fig. 4[5]. 
Table 1 Material property

\begin{tabular}{ccc}
\hline Material & Young modulus (E,GPa) & Poisson's ratio $(\mathrm{V})$ \\
\hline Cortical bone & 14 & $0.30[6]$ \\
Cancellous bone & 3 & $0.30[6]$ \\
Ti6A14V & 110 & $0.35[7]$ \\
Zirconium oxide & 70 & $0.28[7]$ \\
\hline
\end{tabular}

\subsection{Comparison analysis of the capability of bearing the torque between the dental implant system before restoration and the implant system after restoration}

The purpose of the present research was to understand the stresses beard by the dental implant systems before and after restoration by means of stimulating the condition of tightening the central screw during the dental surgery when the accident of central screw fracture is easy to occur; and the central screw or the restorable central screw were acted by the torque. The limit to the torque of the conventional centera screw equal to $30 \mathrm{~N}$.CM was inflicted to the central screw in the dental implant system before restoration in order to perform the overall mechanical analysis; and two torques of 30 N.CM and 60N.CM were inflicted to the restorable central screw of the dental type implant system after restoration, wherein the torque equal to 60N.CM was inflicted to sitimulating the higher original loading and judge whether the whole system may still has the perfect reliability under the condition of enhancing the connection stability. The result was shown in the Fig. 5, the maximum equivalent stress beard by the dental implant system was equal to 828.49MPA, the maximum equivalent stresses beard by the dental implant system were only equal to 263.22MPA and 526.44MPA when inflicting the torque equal to 30 N.CM and equal to 60 N.CM respectively to the restorable central screw o after restoration, and these values were not excess of the yield limit of the material and won't produce the stress rupture'. Therefore, the restoration may increase the original torque, and enhance the connection stability of the dental implant system and may not affect the reliability of the whole system.

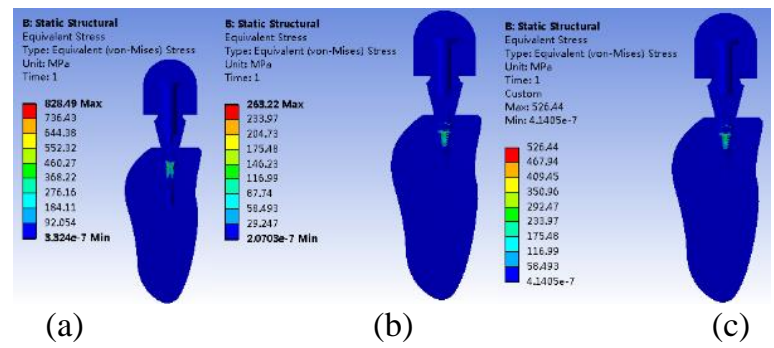

Noted: (a) Result after inflicting a torque equal to 30N.CM to the original dental implant system (b) Result after inflicting a torque equal to 30N.CM to the restorable dental implant system (c) Result after inflicting a torque equal to 60N.CM to the restorable dental implant system

Fig. 5 Force bearing statuses after inflicting the torque to the two dental implant systems

\subsection{Statical analysis and the loading fatigue analysis for the restorable dental implant system and the osseous tissue}

In this study, we stimulated the biomechanics property of the dental implant system and the alveolar bone under the condition of bearing the occlusal force after restoration and the realibility of the dental implant system after restoration and the periphery osseous combination, so that providing all of the contacts among the jaw, the implant and the body were the stationary contacts,[8]. And the lower fractured surface of the alveolar bone was in the fixed constraint. In most of the literatures, a forces equal to $200 \mathrm{~N}$ was loaded on the dental implant system in the actual working conditions in the literature [10 12], and in this article, we stimulated the most possible occlusal force condition by loading a force equal to $300 \mathrm{~N}$ on the restorable dental implant systems under the normal implantation status for the horizontal bone and the status of osteanabrosis up to $2 \mathrm{~mm}$ to perform the biomechanical analysis on the restorable dental implant system. Those were the two status shown in the Fig. 1(b), where the angle between the loading direction of the occlusal force and the vertical direction were equal to $0^{\circ}, 15^{\circ}, 30^{\circ}$ and $45^{\circ}$ respectively under the condition of the bone horizontal implanting 
and the osteanabrosis of $2 \mathrm{~mm}$, and the pretension torsional moment of the restorable central screw was equal to $30 \mathrm{~N} . \mathrm{CM}[10-12]$. We also performed the fatigue analysis at the loading direction of the eocclusal force equal to $30^{\circ}$ which was the recognized occlusive angle for a long time, and the frequency set was equal to $15 \mathrm{~Hz}$, and the acceptance condition should be that the damage to the central screw hasn't occur until bearing $5 \times 10^{6}$ cycles of loading.

The Fig. 6 presented the mechanics equivalent stress results when the angle of loading force on the restorable dental implant system was $30^{\circ}$ in the status of implantation on horizontal bone, and the Fig. 7 shown the result of its fatigue analysis.

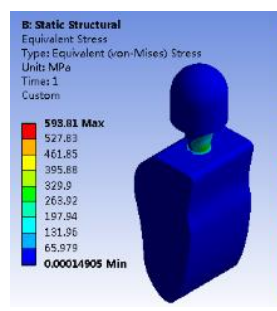

(a)

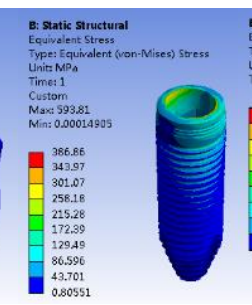

(b)

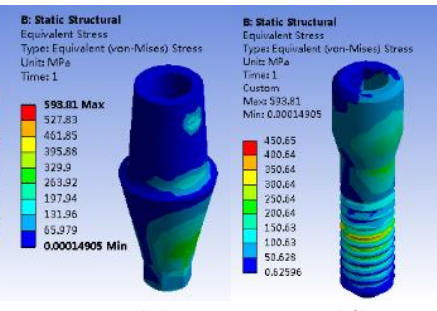

(c)

(d)

(a)Overall mechanical analysis result figure (b) figure for the mechanical analysis result for the body of the implant (c) figure for the mechanical analysis result for the abutment(d) figure for the mechanical analysis result for the central screw

Fig.6 figure of the equivalent stresses beard by the restorable dental implant system and the alveolar bone

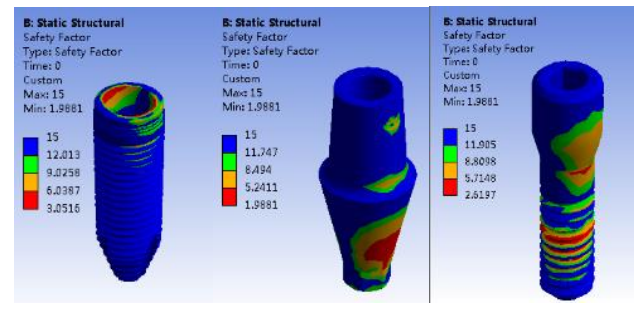

(a)

(b)

(c)

(a) was the figure of the fatigue analysis result for the dental implant body (b) was the figure of the fatigue analysis result for the abutment (c) was the figure of the fatigue analysis result for the central screw.

Fig. 7 figure of the fatigue security coefficient distribution of the restorable dental implant system

\subsection{Result and analysis}

Table 2 was the statistic table for the maximum equivalent stress of every parts in the restorable dental implant system and the alveolar bone when loading a force equal to $300 \mathrm{~N}$ at every loading directions when stimulating implantation in the horizontal bone and in the osteanabrosis up to $2 \mathrm{~mm}$; the table 3 was the minimum safety coefficient comparison table of the dental implant system in the two status when the direction of the loading force was equal to $30^{\circ}$.

Table 2 Statistical table for the maximum equivalent stress of every parts in the implant system (Mpa)

\begin{tabular}{ccccc}
\hline $\begin{array}{c}\text { Status of the } \\
\text { implant }\end{array}$ & $\begin{array}{c}\text { Loading } \\
\text { angle }\end{array}$ & $\begin{array}{c}\text { Implant } \\
\text { body }\end{array}$ & Abutment & $\begin{array}{c}\text { Central } \\
\text { screw }\end{array}$ \\
\hline Bone at & 0 & 88.635 & 616.23 & 439.7 \\
horizontal & 15 & 217.63 & 600.79 & 445.09 \\
& 30 & 187.53 & 593.81 & 450.65 \\
Osteanabrosi & 45 & 529.76 & 598.33 & 455.8 \\
s & 0 & 611.27 & 347.66 & 511.51 \\
& 15 & 692.84 & 372.91 & 623.27 \\
& 30 & 770.47 & 414.44 & 770.96 \\
& 45 & 837.54 & 504.78 & 908.35 \\
\hline
\end{tabular}


Table 3 Table of the minimum safety coefficient comparison between every pats of the implant system.

\begin{tabular}{ccccc}
\hline Loading Force & Loading angle & Dental implant & $\begin{array}{c}\text { Abutmen } \\
\mathrm{t}\end{array}$ & Central screw \\
\hline Bone Horizontal & 30 & 3.0516 & 1.9881 & 2.6197 \\
Osteanabrosis & 30 & 1.5322 & 2.8486 & 1.5313 \\
\hline
\end{tabular}

It was observed from the Fig. 6 that, the external stress beard by the implant body mainly centered at the contact region between the implant bodyand the cortical bone while the internal stress beard by the implant body mainly centered at the connecting cone of the implant body and abutment and the inner screw thread; the crushing stress occurred on the contact surface between the implant body and the abutment leaded to low failure of the contact surface. The upper part of the internal threads may mainly bear the pretightening force, here too high stress occurred, especially the stress centeral occurred at the end of the thread,. The thread junction of the implant body became a risk point of the implant failure. On the horizontal surface of the bone, the equivalent stress at the junction between the implant body and the osseous tissue decreased gradually. This is because the abutment shifting technology adopted in the shoulder part of the implant reduced the low stress level at the junction part between the implant body and the osseous tissue, and thus avoided the decrease of the bone horizontal height caused by the too high stress during the long time service of the implant body. To survey the whole implant system-osseous tissue model, we found that the average stress at the among the stress at the junction part of the implant body and the abutment, and the junction part of the dental implant system and the thread were at a high level, and the stress beard by the osseous tissue was at a low level.

The Fig. 7 revealed that, after the action of $5 \times 10^{6}$ cycles of loading, the dental implant was still in safe status, and the rupture phenomenon caused by the fatigue didn't occur. And the distribution trends of the fatigue safety coefficient was basically the same as that of the stress in the dental implant, the stresses centered at the junction part between the dental implant and the thread of the restorable central screw, the junction part of the dental implant and the abutment, the junction surface between the abutment and the restorable central screw, where the safety coefficients were between 2.0 and 3.0, and thus the dental implant was rather safe.

The table 2 and the table 3 revealed that, the maximum equivalent stress mainly centered on the abutment under the status of implantation in the horizontal bone while the maximum equivalent stress mainly centered on the implant body and the restorable central screw under the status of osteanabrosis, and the value of the stress presented a ascending trend, which illustrated that the osteanabrosis may result in the damage of the implant body and the restorable central screw. In addition, the value of the maximum equivalent stress may change following the change of the loading angle. However, in our actual chewing, the occlusal force at too larger angle won't occur. So that, the dental implant systems under both of the two status were safe when the occlusal force is at the maximum angle equal to $45^{\circ}$, and they may be safe and usable in the actual application. After experiencing stimulation of 5X106 cycles of loading, the safe coefficients were at the same as the maximum equivalent stress, this demonstrated that the dental implant system had perfect system reliability.

\section{Summary}

By means of the above analysis, we may acquire the following conclusion:

1) The restorable dental implant system was designed aiming at the central screw fracture, and based on the analysis on the ANSYS torque, we may know that the restorable central screw was able to bear a higher pretension torsional moment torque of 60 N.CM, and the torsion properties of the restored dental implant system was improved significantly and the restoration method was simple and easy to operate . 
2) With regard to the restorable dental implant system, whether it is under the implantation status on the horizontal bone or implantation status of the osteanabrosis up to $2 \mathrm{~mm}$, the stress beard and fatigue analysis mayn't damage the system when loading a force equal to $300 \mathrm{~N}$ at different angle, and thus the system has perfect reliability.

3) A several of the stress central phenomenon occurred in the analysis, thus the handling of the weakness region in the sharp corner of the thread and the should be considered in the manufacturing process and the optimization and the improvement should be carried out.

\section{Acknowledgments}

This work is supported by the Science and Technology Program of Shenzhen, China (No. JCYJ20140617143643469), Science and Technology Program of Guangzhou (Evaluation of Small and Medium-Sized S \& T Enterprises: No. 201605161031194), Shenzhen Science and Technology Innovation Committee Program (No.CXZZ20140414170821148), Foshan Innovative and Entrepreneurial Research Team Program, China (No. 2014IT100102).

\section{Reference}

[1] Gao Fei,Zhang En-wei,Wei Shi-cheng,Fang Jing. Analysis of static force and fatigue between thread structure of dental implant and contact surface. Journal of Clinical Rehabilitative Tissue Engineering Research, Vol.14 (2010),p:5531-5534.

[2] Zhang Peng,Lin Tian-ci,Huang Da-hong, etc.A comparative study of stress distribution of expandable dental implants and surrounding bone tissues:A three-dimensional finite element analysis. Chinese Journal of Prosthodontics, Vol.16 (2015),p:207-211.

[3] Meng Xiang-yun.A retrospective study of influence of platform switching on peri-implant bone loss. Dalian Medical University.(2013).

[4] Xiao Jian-rui. Optimal selection and experimental study for the retention of expandable implant in osteoporosis. The Fourth Military Medical University.(2011).

[5] Li Yu-jia. Investigation of Fatigue Properties and Fatigue Design Diagram of Titanium alloy Ti-6A1-4V. East China University Of Science And Technology (2014).

[6] Straumann Straumann Product Catalog 2012 (2012)

[7] Lin CL;Wang JC;Kuo YC Numerical simulation on the biomechanical interactions of tooth/implant-supported system under various occlusal forces with rigid/non-rigid connections. Journal of Biomechanics. Vol.39.No.3(2006),p:453-463

[8] Zhao Ji-kui.Finite element analysis of the biomechanics of a dental implant system. Northeastern University.(2012).

[9]Joo-Ho Choi BL. \&Han JS. 2001. Computer Methods in Applied Mechanics and Engineering: Vol. 190(2001), p: 6906.

[10]Mangano FG. \& Cirotti B. \& Sammons RL. \& Mangano C. 2012. Lasers Med Sci: Vol. 27 (2012), p: 1241.

[11]Pirker W. \& Kocher A. Int J Oral Maxillofac Surg: Vol. 38 (2009) , p:1127.

[12]Pirker W. \& Kocher A. Int J Periodontics Restorative Dent: Vol. 31 (2011), p:663.

[13]International Organization for Standardization: ISO 14801-2007 Dentistry - Implants Dynamic fatigue test for endosseous dental implants, IHS under license with ISO (2007). 